\title{
Application of thin-walled dynamics for advanced manufacturing solutions
}

\author{
Zunmin Geng, Keith Ridgway, Sam Turner and Gareth Morgan \\ Advanced Manufacturing Research Centre with Boeing, the University of Sheffield \\ Advanced Manufacturing Park, Wallis way, Catcliffe, Rotherham S60 5TZ, UK \\ z.geng@sheffield.ac.uk; k.ridgway@sheffield.ac.uk .
}

\begin{abstract}
This is a review paper, exampled with several typical applications of thin-walled dynamics for advanced manufacturing solutions. Theoretically, the mechanical and dynamical characteristics of thin-walled structures are discussed. Following this, basic principles of dynamic solutions, including dynamic fixture design for thin-walled structures as well as dynamic treatments of the machining system, are summarized. Several typical applications of these principles for practical manufacturing are illustrated and analyzed, followed with conclusions for the whole paper.
\end{abstract}

\section{Introduction}

Machining of thin-walled components made from difficult-to-machine materials like titanium and heat-resistant-alloy are commonly seen in aerospace industrial, which is one of the most difficult topics for advanced manufacturing technology [1-3]. Among several key influential factors, the unstable dynamic performance of thin-walled structure plays an important role in exciting the workpiece through the machining process, which causes machining problems like machining-chatter, unsatisfied surface-finish and precision, work-piece distortion and excessive in-workshop noise, etc. Different thin-walled structures demonstrate different dynamical performances which could require different fixtures and/or machining methodologies to reach a stable machining process. Based on these understandings, this paper reviews several successful applications of thin-walled dynamics for advanced manufacturing solutions. Theoretically, the mechanical and dynamical characteristics of thin-walled structures are discussed. Following this, basic principles of dynamic solutions, including dynamic fixture design for thin-walled components as well as dynamic treatments of the machining system, are summarized. Several typical applications of these principles for practical manufacturing are illustrated and analyzed, followed with conclusions for the whole paper.

Figure-1 illustrates an aero-engine construction, in which more than $90 \%$ parts can be classified as thin-walled structures: the blades can be classified as thin-walled-asymmetric-open-components and the engine-casing can be classified as thin-walled-axisymmetric-closed-components, etc. It is therefore necessary to investigate the different dynamic characteristics from different types of thin-walled components and then conclude several basic principles for reasonably treating these thin-walled parts stably through the machining process.

\section{Dynamic characteristics of thin-walled structures}


2.1. Definitions by structural mechanics and dynamics

There are different definitions on thin-walled structures from different theories. Generally, the following two respectively based on Solid Mechanics and Structural Dynamics can explain the phenomena occurred in manufacturing area.
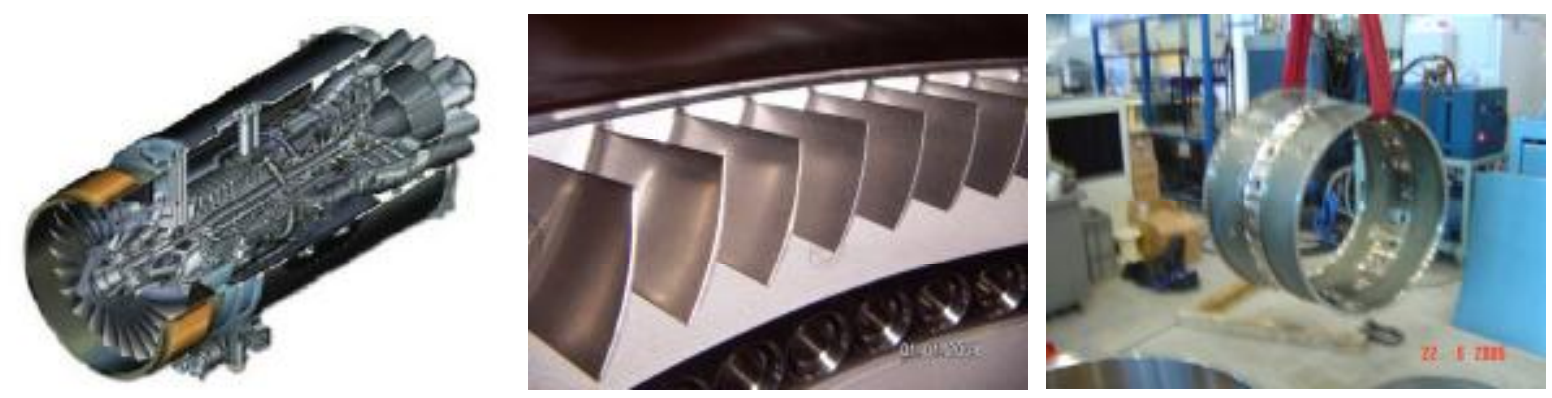

(a). More than 90\% thin-walled parts. (b). Asymmetric-open-blades. (c). Axisymmetric-closed-casing.

Figure-1. A typical aero-engine construction

\subsubsection{Definition by Solid-Mechanics}

According to classical solid mechanics theory [4-5], shell and plate-like structures can be classified into the followings:

(1) Very-thick-walled structures: Three-dimensional stressed, the so-called solid-structures.

(2) Thick-walled structures: Stretching, bending and higher order shear stressed.

(3) Moderate-thick-walled structures: Stretching, bending and first-order shear stressed.

(4) Thin-walled structures: Mainly stretching and bending stressed, with shear stress negligible.

(5) Very-thin-walled structures: Dominated by stretching-effects, the so-called membranes.

There is also a conventional classification of thin-walled structures in the engineering areas: Structurecurvature-to-wall-thickness-ratio $\geq 20$, with only stretching and bending stresses to be considered and transverse-shear-stress negligible.

\subsubsection{Definition by Structural Dynamics}

According to classical structural dynamics theory [5-9], a thin-walled structure can be identified mainly based on the followings:

(1) Low structural stability and rigidity (Buckling [5-6]).

(2) Structure-borne wave-propagation and acoustic-reverberations [6-9].

Another theoretic issue must be emphasized is that most thin-walled structures are subject to continuous system modelling, identifications and solutions, through structure-borne acoustics and/or Statistic-Energy-Analysis (SEA) approaches [7-12], for a better theoretical understanding and practical engineering applications.

\subsection{Dynamic characteristics of typical thin-walled structures}

\subsubsection{Example-1 with open and/or closed thin-walled parts: Static/Dynamic buckling}

Figure-2 shows a typical test with the classical buckling theory [4-5], which is applicable both for thin-walled open structures like a slender blade and closed structures like an engine-casing shown in the Figure. Within a pre-buckling-limit which is determined by the structure's mechanical properties [4], a linear relationship between the testing-load and displacement is roughly maintained. When the testing-load reaches up to the buckling-limit, a slight transverse perturbation could induce the structure 
into a full collapse, so as to loss a minimum structural rigidity which supports the component for the relevant machining work. Mainly from this consideration, adaptive and/or active design of fixtures is becoming a popular topic in the area [1-2, 15-18].
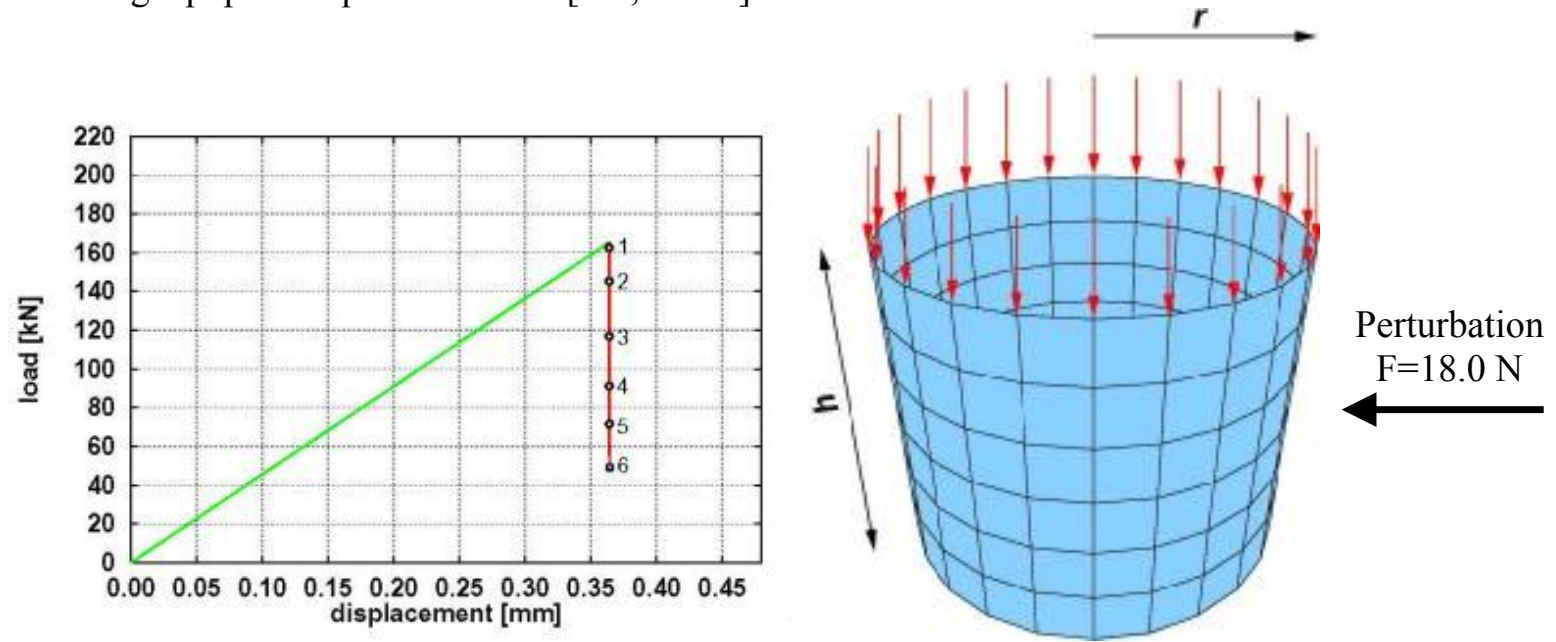

Figure-2. A thin-walled cylinder, stable within the buckling-limit and buckling formation with a minor perturbation at the buckling-limit

2.2.2. Example-2 with 2-D and 3-D axisymmetric thin-walled parts: Multi-eigen induced instability. Figure-3 shows a 3-Dimensional axisymmetric thin-walled component, with identified dynamicresponses both in time and frequency domains. According to classical theory of structure-borne acoustics and vibration [5-9], there will be a multi-eigen (structural resonances, triple-eigens from 3-D and double-eigens from 2-D structures) problem which induces modulated vibration and travellingwave-propagation around the disc, so as to put the work-piece under an unstable dynamic condition through the machining process. A practical unstable machining example is shown in Figure-4.
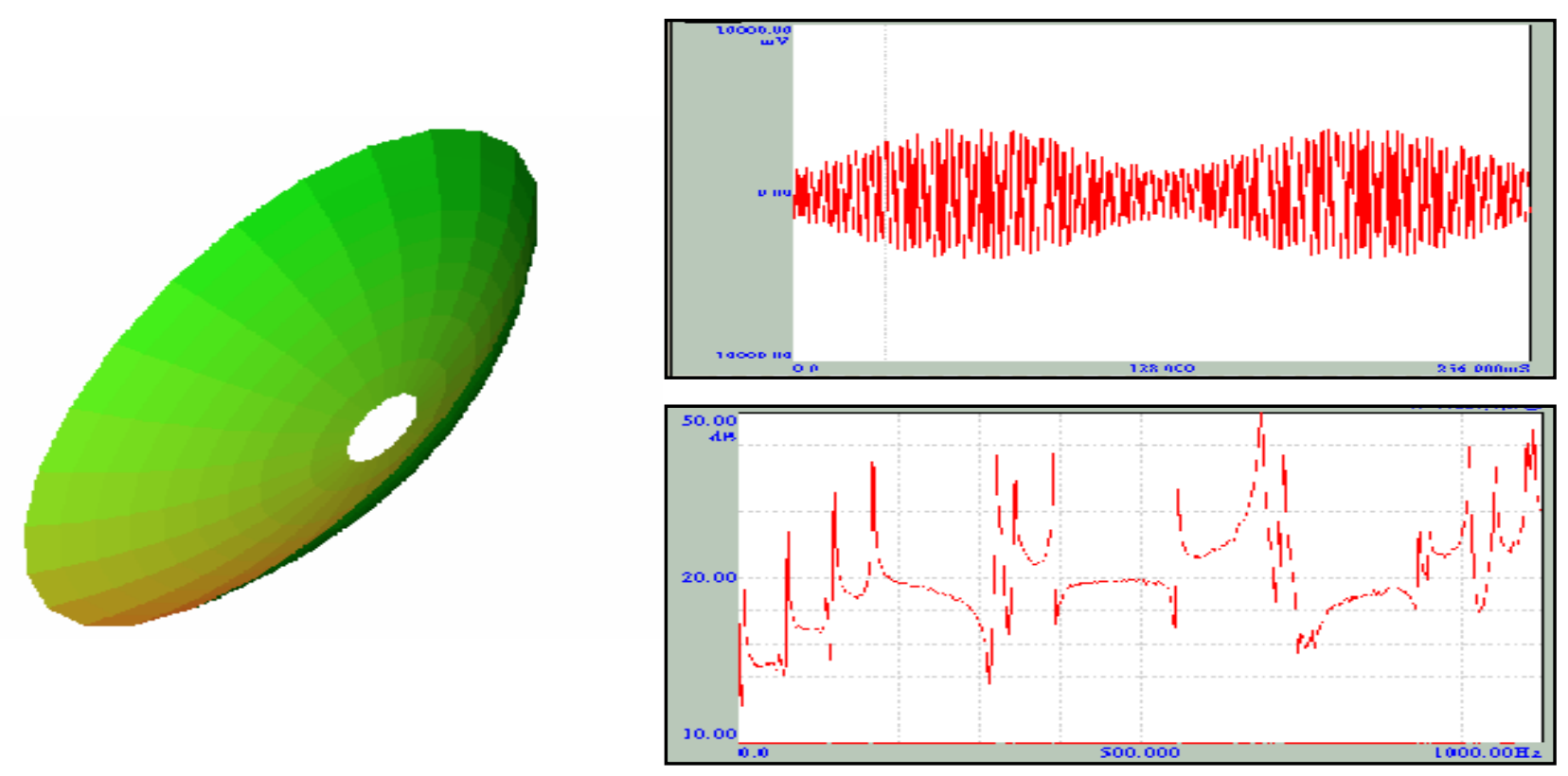

Figure-3. Triple-eigen induced travelling-wave propagation along a 3-D axisymmetric part 


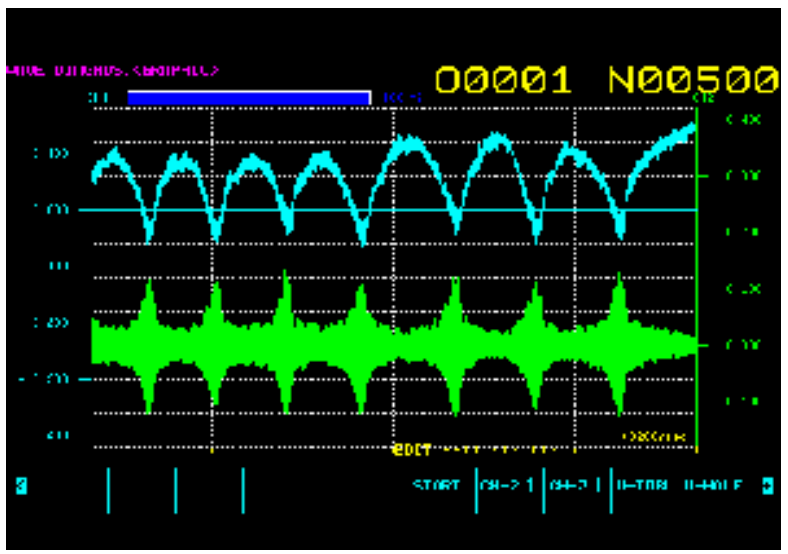

(a). Machine-tool power fluctuation ( $\mathrm{kW}, 0-3.2 \mathrm{sec})$

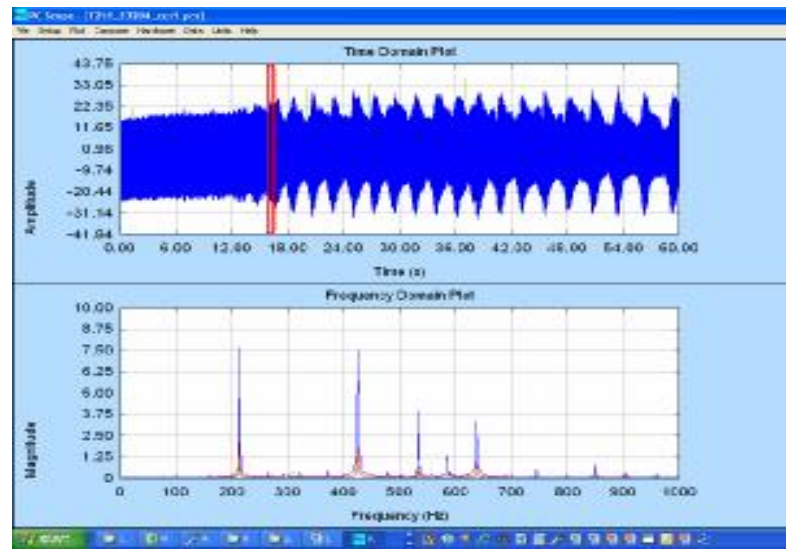

(b). Modulated vibration $\left(\mathrm{m} / \mathrm{s}^{2}, 0-60 \mathrm{Sec}\right)$

Figure-4. Double-eigen induced machining-instability in grinding a 2-D axisymmetric disc-part

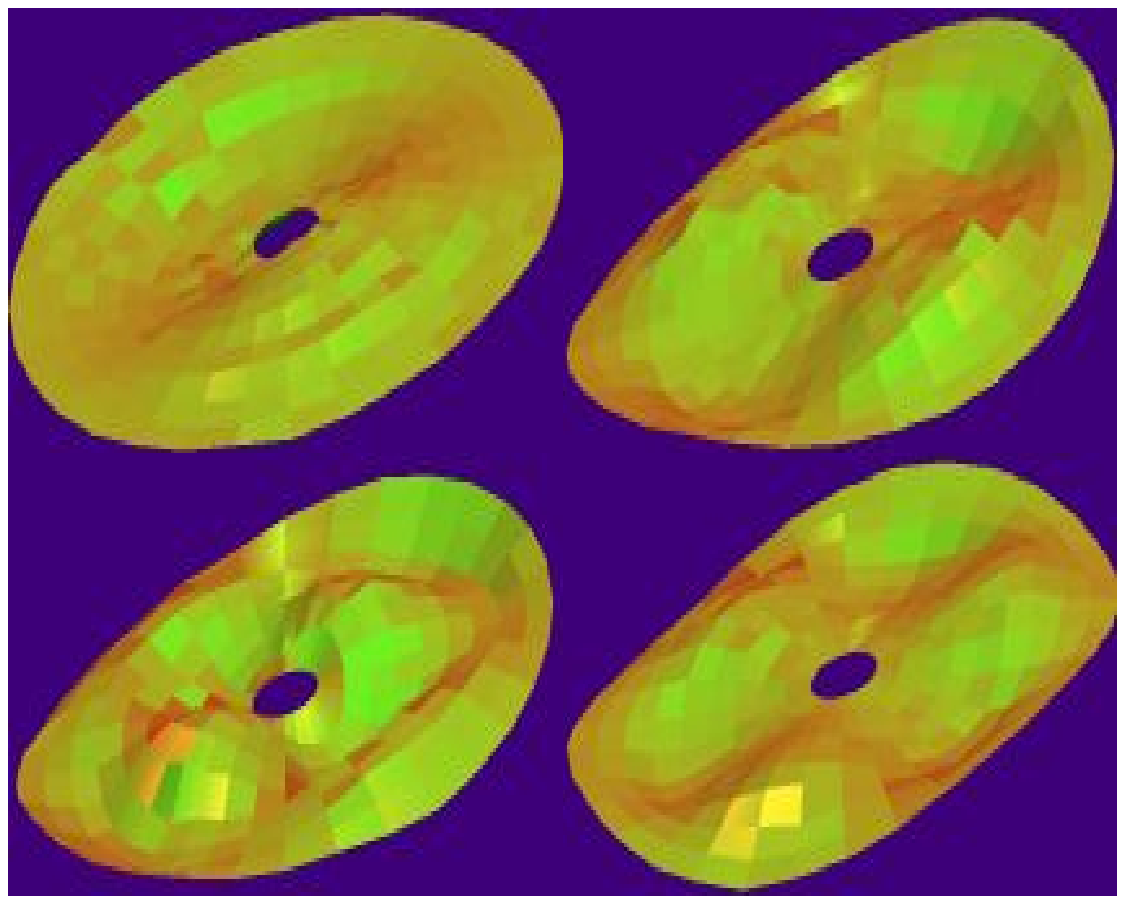

Figure-5. Double-eigen induced travelling-wave propagation around an axisymmetric 2-D disc and working-mechanism of ultrasonic-motors

Figure-5 and equations (1) to (4) explain the happening of multi-eigen problem with random travelling-wave-propagation in unstable machining processes, and with controllable travelling-wavepropagation as the working-mechanism of ultrasonic motors [9-10], as follows:

Theoretically analyzing an annual thin-walled plate with axisymmetric boundary conditions, in a continuous-structural-modelling procedure, its out-of-plane Eigen solutions can be expressed as:

$$
w_{k n}(r, \theta)=A_{k n} \cdot R_{k n}(r) \cdot \begin{cases}\cos (k \theta) & k=0,1,2, \mathrm{~K} \\ \sin (n \theta) & n=1,2,3, \mathrm{~K}\end{cases}
$$


Where $R_{k n}(r)$ is Bessel function and $A_{k n}$ are modal constants determined by initial boundary conditions in two nodal directions $k$ and $n$. Considering the cosine and $\sin \operatorname{modes} w_{c}$ and $w_{s}$ corresponding to the same Eigen-frequency $\omega_{k n}$ of multiplicity-2 to be linearly independent, then equation (1) can be further derived as:

$$
\begin{aligned}
w(r, \theta, t, \varphi) & =w_{c}+w_{s} \\
& =A R(r) \cos (k \theta) \cos (\omega t)+B R(r) \sin (k \theta) \sin (\omega t+\varphi)
\end{aligned}
$$

Or, in another form:

$$
w(r, \theta, t, \varphi)=\frac{1}{2} R(r)\left[\begin{array}{l}
(A+B \cos \varphi) \cos (k \theta-\omega t) \\
+(A-B \cos \varphi) \cos (k \theta+\omega t)+2 B \sin \varphi \sin k \theta \cos \omega t
\end{array}\right]
$$

The first and second terms of equation (3) correspond respectively to a bending wave travelling in the positive (anti-clockwise) and negative (clockwise) $\theta$ directions, and the third term represents an unmovable standing-wave-oscillation (dominant modes of the structural vibration). This equation demonstrates a random combination of standing-wave-oscillation and uncontrollable travelling-wavepropagations around the disc in clockwise and anti-clockwise directions, which frequently happens in the unstable machining process as shown in Figure- 4.

Further controlling the model's initial and boundary conditions to make $\varphi=0$ and $A=B$, then

$$
w(r, \theta, t)=A R(r) \cos (k \theta-\omega t)
$$

Equation (4) clearly shows that, by controlling the two independent eigen-modes from the doubleeigen solution in phase with the same amplitude, one can generate a travelling-wave-propagation rotating around the disc in a controllable direction, clockwise or anti-clockwise, with a controllable rotating-speed (propagation velocity) $\omega / k$. This is the working-mechanism of ultrasonic-motors [10], as illustrated in Figure-5.

\subsubsection{Example-3 with 1-D slender open parts: Standing-wave-scillation induced machining noise.}

Figure-6 demonstrates a 1-Dimensional slender thin-walled component with finite length, coupled with a theoretic model from Professor M. J. Brennan which describes the standing-wave oscillation effects inside the part [11]. This model explains why heavy noise ( $>95 \mathrm{~dB})$ generates from machining this slender part, also recommends a discrete vibration neutralizer to take the flexural-standing-waveoscillation under control.

According to classical theory of structure-borne-acoustics [8-11], another potential solution to reduce the machining-induced noise could lie in the possibility of transferring the standing-wave-oscillation inside the finite-length part into a travelling-wave-propagation descending into a man-made infinity.

\subsubsection{Example-4 with 3-D closed thin-walled shell parts: Complex acoustic reverberation and mode congestion}

Figure-7 shows a freely suspended engine casing with its modal testing results. This component is a typical 3-Dimensional closed thin-walled structure and its dynamical characteristic is too complex to be analytically described with the above-mentioned multi-eigen modelling approach. However, several 
identification rules can still be summarized from the classical theory of structure-borne acoustics [811] and SEA [12], as detailed below.
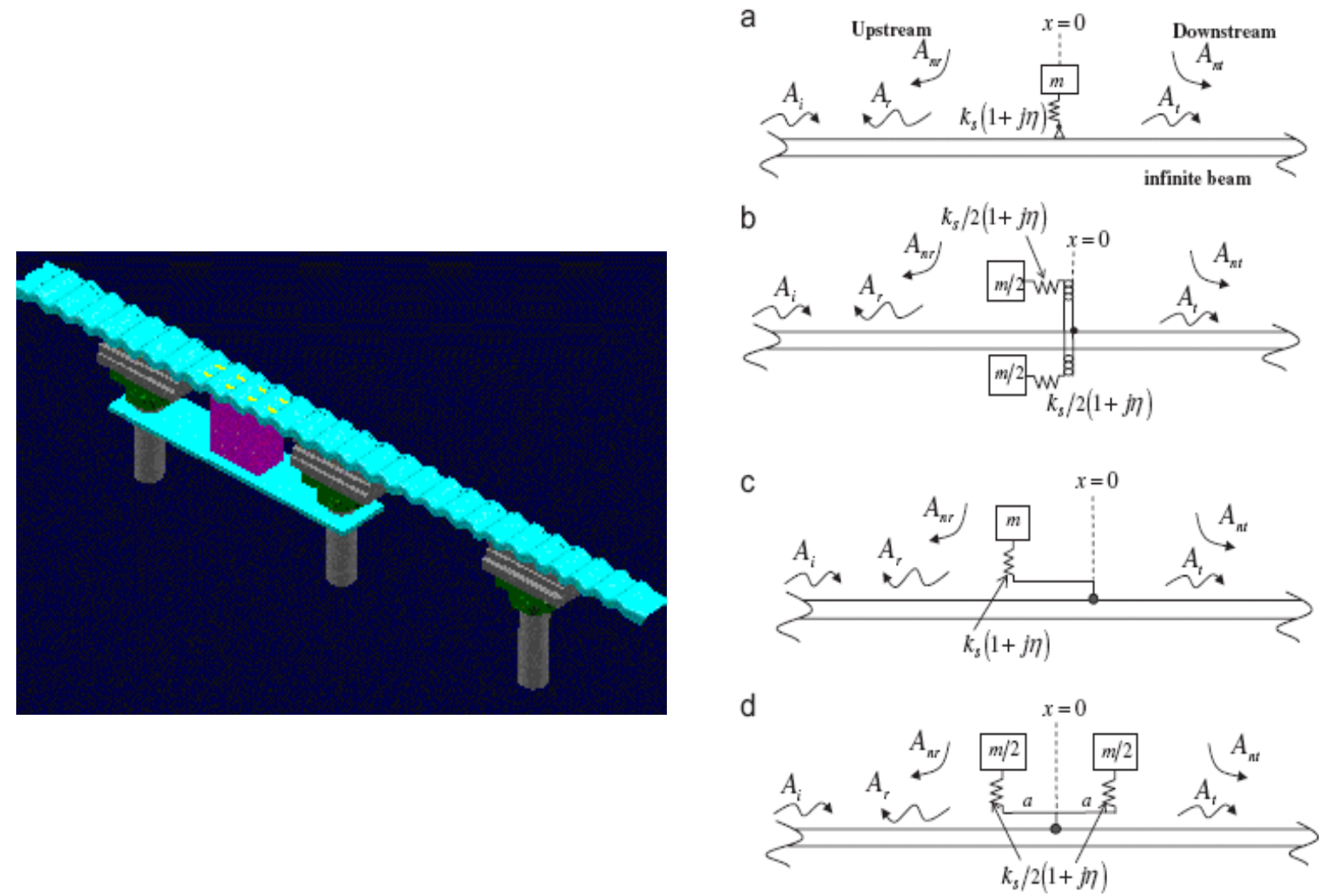

Figure-6. A noisy thin-walled part in machining-position and its wave-propagation modes
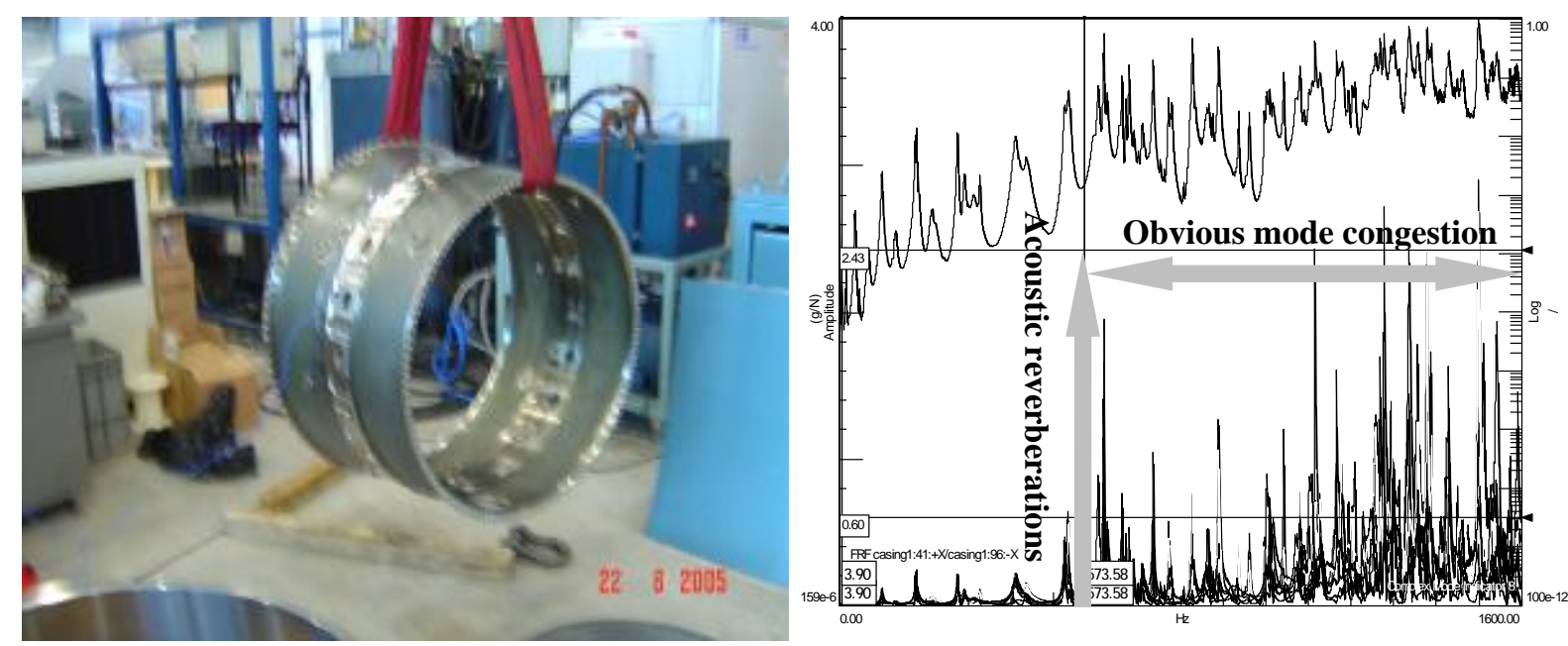

(a). A freely suspended engine-casing for modal test.

(b). Identified CMIF and mode-ensemble

Figure-7. A freely suspended engine-casing and its statistical structural responses

(1) Heavy structure-borne acoustic reverberations spread over a wide frequency range.

Figure-7(b) shows an experimentally identified mode-ensemble of the freely suspended enginecasing as shown in Figure-7(a), the lower cluster of FRFs in the Figure as described in Equation (5). Very clearly, in the low-frequency-range from $0.0 \mathrm{~Hz}$ to $573.00 \mathrm{~Hz}$, repeatable and stable 
vibration modes are identifiable. Beyond this frequency range $(>570.00 \mathrm{~Hz})$, the identified resonant frequency at one point is quite different and shifting from that identified at another point. This random local-mode shifting and moving is an obvious evidence of structure-borne acoustic reverberations [8-12], which incurs the structure dynamically unstable through the relevant machining processes.

(2) Obvious mode-congestion identifiable from mode-ensemble and CMIF

The upper part of Figure-7(b) (single curve) is the further calculated Complex-Mode-IndicatorFunction (CMIF) from the experimentally measured mode-ensemble [9, 12-13]. According to the theory of MA \& SEA [9-13], the identified mode-ensemble, or the FRF matrix of the structure with $N_{o}$ (Output: Structural vibration response in $\mathrm{m} / \mathrm{s}^{2}$ ) and $N_{i}$ (Input: Excitation-force in N) degrees of freedom can be expressed as follows:

$$
[H(\omega)]=\sum_{r=1}^{2 N}\{\Phi\}_{r}\left[\begin{array}{c}
Q_{r} \\
\omega-\lambda_{r}
\end{array}\right]\{L\}_{r}^{T}=[\Phi]\left[\begin{array}{c}
Q_{r} \\
\omega-\lambda_{r}
\end{array}\right][L]^{T}
$$

Where: $[H(\omega)]=[H(\omega)]_{N_{i} \times N_{o}}$ is the FRF matrix of size $N_{i}$ by $N_{o}$.

$[\Phi]$ is the mode shape matrix of size $N_{o}$ by $2 N$ (N: mode numbers to be identified).

$Q_{r}$ is the scaling factor for the rth mode.

$\lambda_{r}$ is the system pole value for the rth mode, and

$[L]^{T}$ is the transposed modal participation factor matrix of size $N_{i}$ by $2 N$ (N: mode numbers to be identified).

Further taking the singular value decomposition of the FRF matrix will result in:

$$
[H]=[U][S][V]^{T}
$$

Where: $[U]$ is the left singular matrix corresponding to the matrix of mode shapes.

$[S]$ is the diagonal singular value matrix, and

$[V]$ is the right singular matrix corresponding to the matrix of mode shapes.

From Equation (6), the Complex-Mode-Indicator-Function (CMIF) can be calculated as follows [13]:

$$
\begin{aligned}
& {[H]^{T}[H]=[V][S]^{2}[V]^{T}} \\
& \operatorname{CMIF}_{k}(\omega)=u_{k}(\omega)=s_{k}(\omega)^{2}, \quad k=1,2,3, \mathrm{~K}, N_{i}
\end{aligned}
$$

Where: $u_{k}(\omega)$ is the kth eigenvalue of the normal FRF matrix, and $s_{k}(\omega)$ is the kth singular value of the FRF matrix, at frequency $\omega$.

It is noticed that, the full mode shape matrix $[H(\omega)]=[H(\omega)]_{N_{i} \times N_{o}}$ has been introduced into the calculation of CMIF at every spectral line, and the singular value decomposition procedure described in Equation (5) to (7) automatically transforms the initial linear-modal-expression in Equation (5) into an nonlinear power, or Statistical-Energy-Analysis expression [12], which is a continuous system 
modelling procedure and very suitable in analyzing or describing the dynamical characteristics of thinwalled structural systems [8-13].

An important dynamic characteristic of complex thin-walled structures can be found from the further calculated CMIF as shown in Figure-7(b) (single curve), that is, within a very low frequency range from $0.00 \mathrm{~Hz}$ to $573.00 \mathrm{~Hz}$, repeatable and stable rigid-structure modes are identifiable which means a solid-structural vibration solution (increase of structural stiffness, etc) still achievable in improving the fixture and machining process, while in the higher frequency range ( $>573.00 \mathrm{~Hz}$ for this component), an obvious mode congestion phenomenon is noticed which means too many unstable vibration modes congested together to be distinguished and identified for a realistic vibration solution. In an academic sense, this frequency border-line $(573.00 \mathrm{~Hz}$ for this component) represents the division of two research areas, structural vibration and structure-borne acoustics. In the higher frequency range beyond the border-line, effective structure-borne-acoustic solutions such as acoustic-energyabsorption and/or global-damping application via SEA approach should be taken into serious considerations in improving the fixture and machining processes of the thin-walled components.

\subsubsection{Example-5 with high profile-to-wall-thickness-ratio parts: Continuous modelling \& solutions} for continuous structures

In explaining the dynamics of complex thin-walled structures as shown in Figure-7, the MA (ModalAnalysis) and SEA (Statistic-Energy-Analysis) concepts as described in Equations (5) to (8) are introduced. These methods are parts of the continuous modelling \& designing solutions for continuous structures (not the conventionally simplified lump-mass models). Another typical application example for advanced manufacturing solution is illustrated below:

Figure-8(a) shows a large-size tombstone fixture for advanced manufacturing (profile-to-wallthickness-ratio $>50$ ) and its lowest 6 vibration modes from FE simulations. In order to make the fixture dynamically strong enough, in support of a stable machining work on the attached work-piece, Tuned-Mass-Damper (TMD) could be a solution, according to the classical theory from J. P. Den Hartog [14].

However, the classical theory of TMD [14] is based on a simplification assumption of Single-DegreeOf-Freedom (SDOF) Lumped-Mass system. For the Multi-Degree-Of-Freedom (MDOF) continuous structure shown in Figure-8(a), a continuous-system-modelling-approach via SEA [12] as shown in Figure-8(b) could be more effective and realistic for engineering applications.

Assuming a series of discrete dynamic-resonators elastically installed on a flexible thin-walled component, through a specifically designed connecting-end-coupling as shown in Figure-8(b), then the dynamic input-output relations of the system can be expressed as:

Discrete dynamic-resonators:

$$
F_{1}=\left[F_{11}, F_{12}, \mathrm{~K}, F_{1 n}\right]^{T}, \quad V_{1}=\left[V_{11}, V_{12}, \mathrm{~K}, V_{1 n}\right]^{T}
$$

Couplings of resonator to main structure: $F_{2}=\left[F_{21}, F_{22}, \mathrm{~K}, F_{2 n}\right]^{T}, \quad V_{2}=\left[V_{21}, V_{22}, \mathrm{~K}, V_{2 n}\right]^{T}$

Flexible thin-walled main structure: $\quad F_{3}=\left[F_{31}, F_{32}, \mathrm{~K}, F_{3 n}\right]^{T}, \quad V_{3}=\left[V_{31}, V_{32}, \mathrm{~K}, V_{3 n}\right]^{T}$

Where: $\quad n$ is the numbers of discrete-dynamic-resonators or mode-numbers to be controlled.

$F_{i}=\left[F_{i 1}, F_{i 2}, \mathrm{~K}, F_{i n}\right]^{T}$ is the excitation-force-vector in RMS, $i=1,2,3$.

$V_{i}=\left[V_{i 1}, V_{i 2}, \mathrm{~K}, V_{i n}\right]^{T}$ is the vibration-response-vector in RMS, velocity $(\mathrm{m} / \mathrm{s}), i=1,2,3$. 

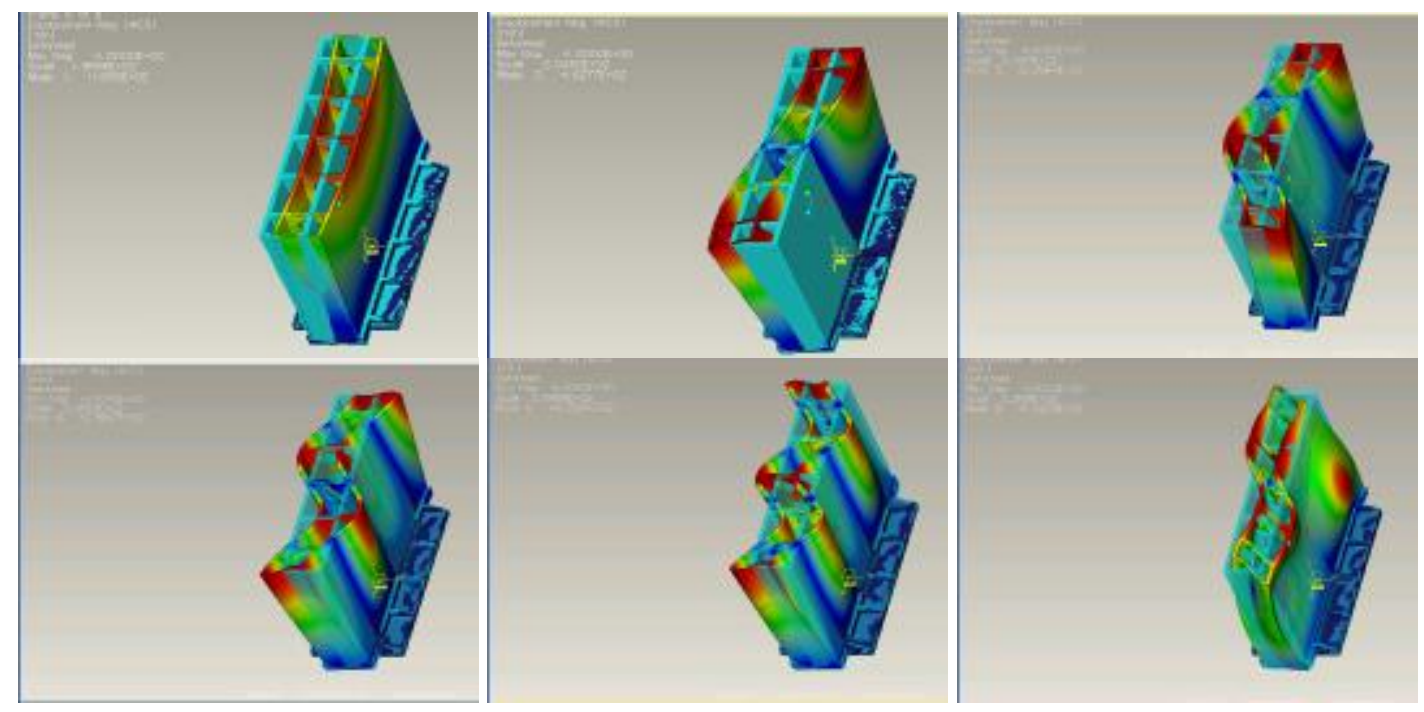

(a). The lowest 6 vibration modes of a large-size continuous structure

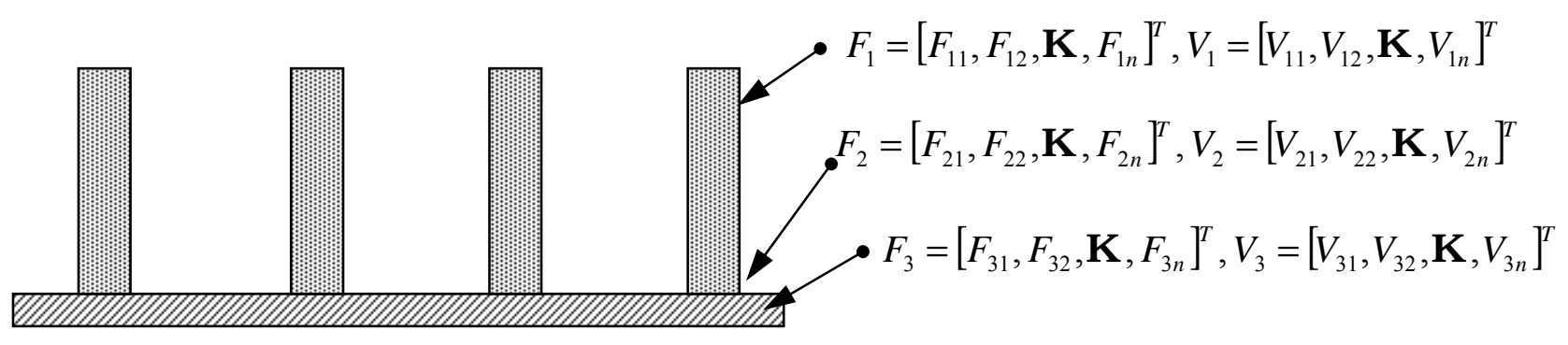

(b). A schematic of SEA modeling procedure for continuous structures

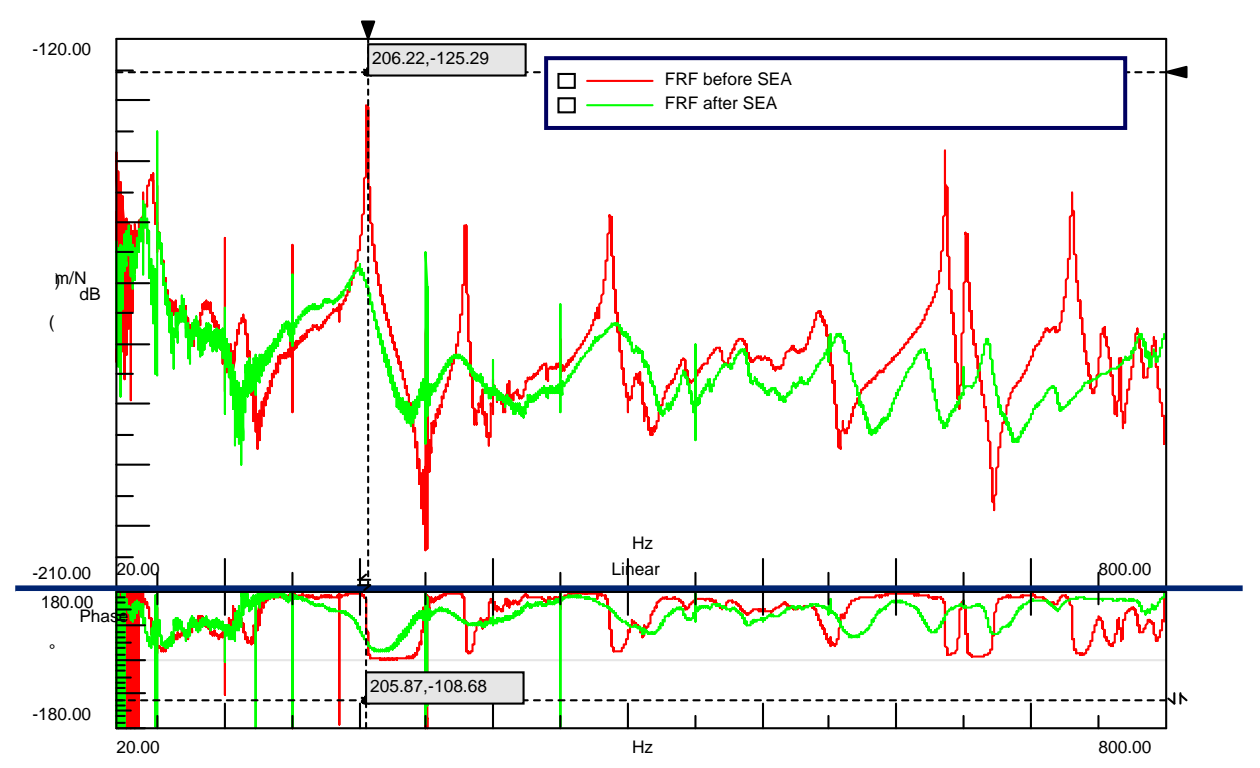

(c). Dynamic response before (red-line) and after (yellow-line) a SEA solution

Figure-8. An application example of continuous-system-modelling \& SEA solutions 
Further correlating the system's inputs and outputs, then the power-flow of the flexible system can be expressed as:

$$
P=\frac{1}{2} \operatorname{Re}\left\{\left\{F^{*}\right\}^{T}\{V\}\right\}=\frac{1}{2} \operatorname{Re}\left\{\left\{F^{*}\right\}^{T}[H]\{F\}\right\}=\frac{1}{2} \operatorname{Re}\left\{\left\{\left\{F_{1}^{*}\right\}^{T},\left\{F_{2}^{*}\right\}^{T},\left\{F_{3}^{*}\right\}^{T}\right\}\left\{\begin{array}{l}
V_{1} \\
V_{2} \\
V_{3}
\end{array}\right\}\right\}
$$

Equation (9-4) represents a continuous model for a flexible continuous system shown in Figure-8 (a). Taking this equation as a target-function for structure optimization [12-13, 19], the structure's dynamic behavior can be, and has been significantly improved, with the FRF-amplitude suppressed down to 20 to $40 \mathrm{~dB}$ through a wide frequency range (a lumped-mass-solution only for a single frequency), as shown in Figure-8-(c).

\section{Dynamic fixture solutions for thin-walled structures}

From the above discussions, several basic principles can be summarized on how to reasonably design a dynamic fixture solution for the machining of thin-walled structures in the advanced manufacturing area, with some typical examples as below:

\subsection{Principle-1: Dynamic design for dynamic use.}

Dynamic fixture solution is a key-concept in reasonable support of a thin-walled component through the machining process. Figure-9 illustrates the importance of this concept through an unsuccessful static fixture solution for thin-walled engine-casing application.

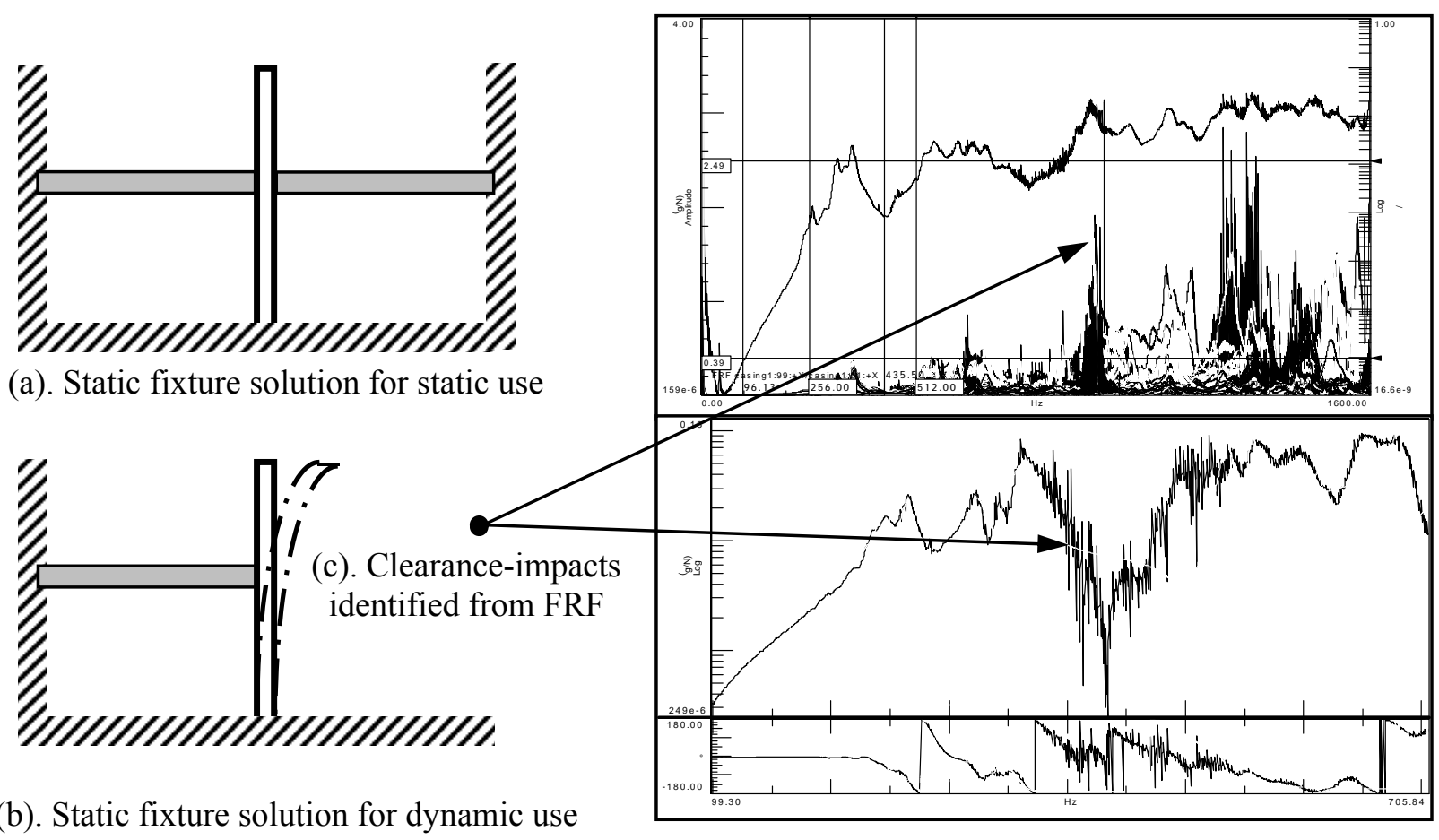

Figure-9. An application example of static fixture solution for dynamic use

Figure-9(a) demonstrates a traditional mechanical fixture solution which statically clamps on both sides of a thin-walled beam for tip-machining. This design is based on the traditional structural 
mechanics theory [4] which is a successful application and can be concluded as 'static fixture solution for static use'.

Figure-9(b) demonstrates the same mechanical fixture which statically supports on one side of the thin-walled beam for tip and surface machining on the other side. This static fixture is obviously unable to stably hold the work-piece through the dynamic machining process. Under the dynamic machining-force excitation, this thin-walled part is bouncing against the supporter and making the machining process even more unstable. A practical FRF test is carried out with a thin-walled engine supported by a similar static fixture, as shown in Figure-9(c), where heavy clearance-induced impacts are identified from the testing results. This unsuccessful fixture solution can be concluded as 'static fixture solution for dynamic use'.

In the following contents, a successful 'dynamic fixture design for dynamic use' example will be further discussed.

\subsection{Principle-2: Continuous modelling \& solutions for continuous structures.}

As theoretically explained and practically exampled in Equations (8), (9), figure-6, 7 and 8, taking most thin-walled components as continuous structures and seeking a dynamic fixture solution via continuous system modeling, identification and designing procedures will be another key-concept. Statistic-Energy-Analysis [12] and Adaptive/Active fixture design [15-18] for an improved global performance as introduced below will be a strong supporting evidence of this concept.

3.3. Principle-3: Adaptive/Active supports for a higher structural stability \& rigidity.

Adaptive/active global support with damping-capabilities is an ideal fixture solution for thin-walled components [15-18]. Where an accurate structural dynamics model is achievable, the active fixture and active-control-algorithm can be designed for the practical applications [16-18]. In most industrial applications, however, the passive global-supporting-fixture with adaptive capabilities is playing a more and more important role for thin-walled structures machining $[15,18]$.

Figure-10 shows a patented pneumatic-adaptive-global-supporting-fixture for the machining of thinwalled engine casings [15]. Comparing the MA testing results of the same part with and without the support of this adaptive-fixture, as respectively shown in Figure-7 and -10, the low dynamic-stability and heavy acoustic-reverberation caused vibration-mode-congestion phenomenon as shown in Figure7(b) has been much improved into a sparse-distribution of vibration-modes, as shown in Figure-10(b). This represents a significant improvement on the casing's dynamic stability \& rigidity, in support of it through the relevant machining process.

Figure-11 presents another strong evidence on the effectiveness of this adaptive fixture for thin-walled structures. By applying a static-load from a load-cell on the casing $(\mathrm{kN})$, the corresponding casingdisplacement $(\mathrm{mm})$ is recorded and the averaged linear-supporting-stiffness $(\mathrm{kN} / \mathrm{mm})$ is calculated, denoted as $\mathrm{K} 1, \mathrm{~K} 2$ and $\mathrm{K} 3$ with pneumatic-pressure to be $0.0,1.0$ and 2.0 Bars. Apparently, $K^{3 / K} 1 \approx 3.40$ represents a significant increase of global-supporting-stiffness from the adaptive fixture on to the thin-walled casing to be machined.

3.4. Principle-4: Adaptive/Active damping for a higher structural \& machining stability.

The patented adaptive fixture [15] includes a pneumatic element which supplies a global-adaptivesupport onto the thin-walled part. Also from this adaptive capability, the pneumatic element applies a sophisticated but very effective adaptive damping on the thin-walls, mainly from the energyabsorption-effects of the pneumatic-cavities [19]. 


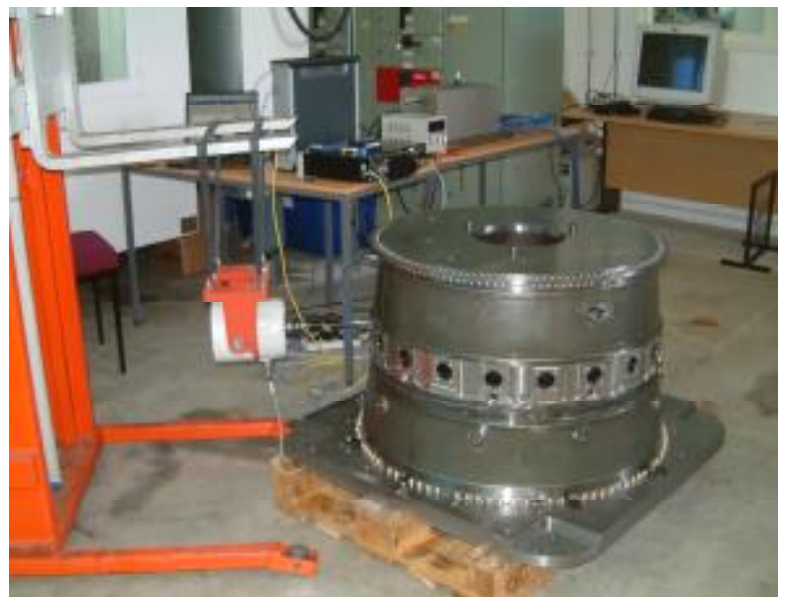

(a). Adaptive-fixture in an engine-casing for MA test.

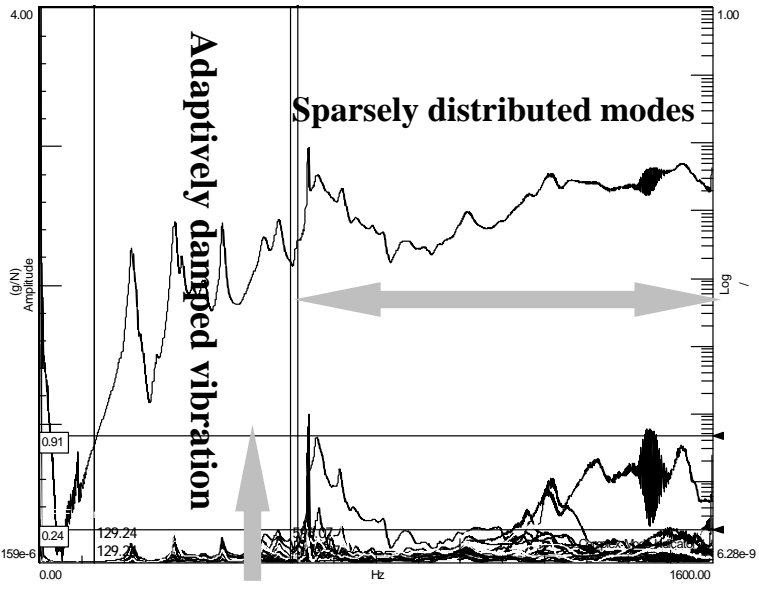

(b). Identified CMIF and mode-ensemble

Figure-10. A Patented [15] adaptive fixture for engine-casing machining

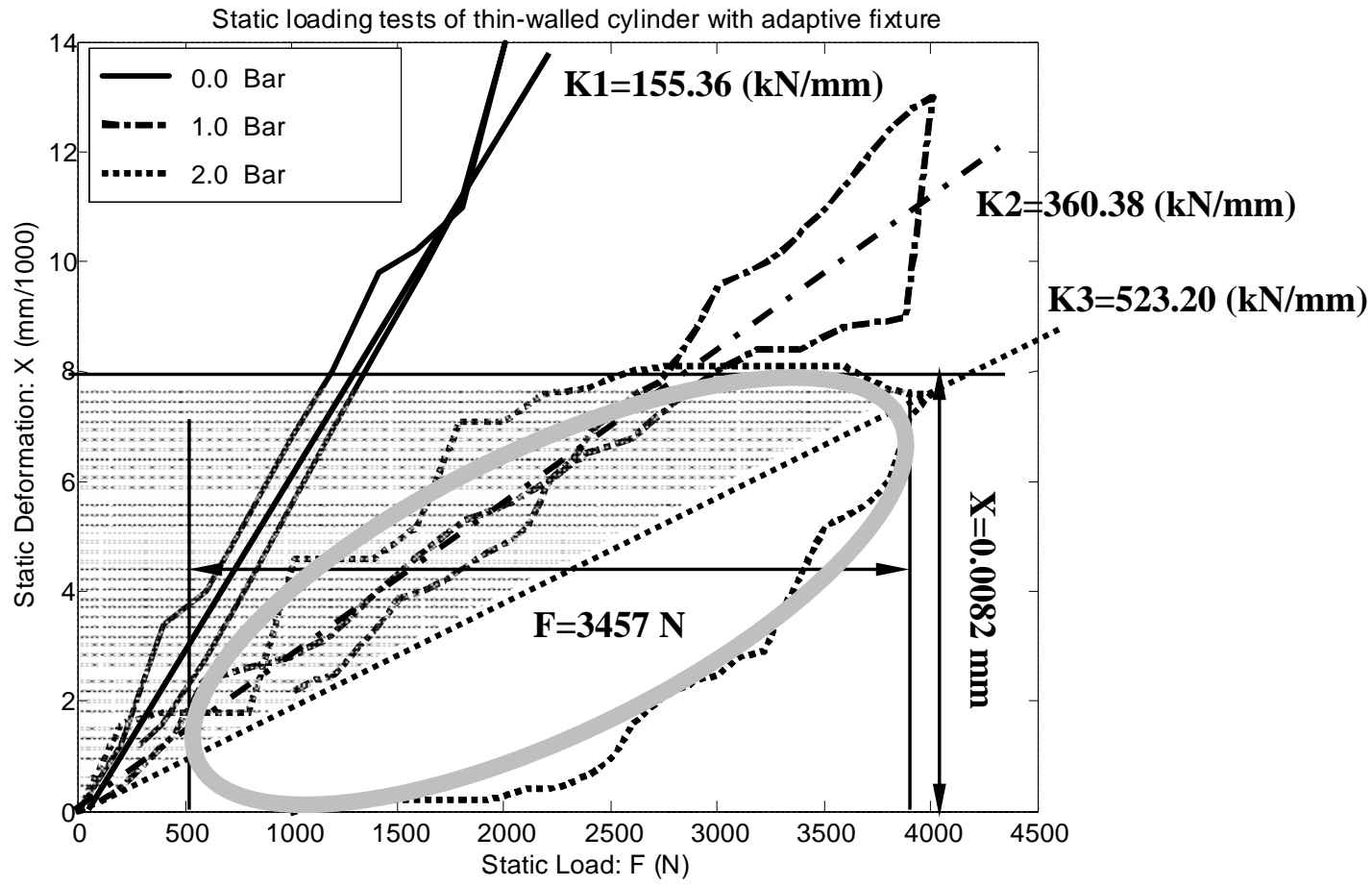

Figure-11. Loading-test on the engine-casing with adaptive fixture installed

As the authors investigated [19], accurate measurement, identification and modelling of the sophisticated adaptive damping effects from a pneumatic-cavity have been a very difficult and advanced topic. According to classic damping theory [4-9, 19-22], when damping is small (losscoefficient $\eta<0.01$ ) and the whole structure is excited near to resonance, a convenient correlation between different measures of damping effects can be estimated from a FRF and/or loading tests, as follows:

$$
\eta \approx \frac{D}{2 \pi} \approx 2 \xi \approx \frac{\delta}{\pi} \approx \tan \psi \approx \frac{1}{Q}
$$


Where: $\xi$ is the damping ratio, measurable from FRFs.

$\delta$ is the logarithmic decrement, measurable from time-domain impulse-responses.

$\eta$ is the loss-coefficient, $D$ is the proportional-energy-loss-per-cycle, $\psi$ is the loss-angle and $\mathrm{Q}$ is the quality-factor, measurable from static and/or dynamic loading-tests.

When damping is large (loss-coefficient $\eta>0.01$ ) and beyond the generally accepted viscous and/or proportional damping assumptions [19-22], the damping measures as correlated in equation (10) will be not equivalent any more. A practical and reliable measure is assessing the hysteresis-loop from a static or dynamic loading test, with outputs as $\eta, D, \psi$ and $\mathrm{Q}$, etc $[15,21]$.

Figure-11 demonstrates an incremental loading-and-unloading testing-loop on the engine-casing with the adaptive pneumatic fixture in-support. It is noticed that, when the pneumatic-pressure is set respectively at 0.0 Bar and 1.0 Bar, the adaptive-damping-effects on the casing are mildly small, when the pneumatic-pressure reaches up to 2.0 Bar, however, a very well developed hysteresis-loop forms from the testing process which means a significant adaptive damping applied on the casing from the fixture. From this practically measured hysteresis-loop, the Loss-Coefficient $\eta$ can be calculated as below:

As shown in Figure-11, the left-side triangle-enclosed shadow-area represents the elastic-energy stored from the loading-test process, which can be approximately expressed as:

$$
U=\int_{0}^{F_{\max }} F d X \approx \frac{1}{2} K_{3} X_{\max }^{2}
$$

The hysteresis-loop enclosed area (Elliptic-enclosure) represents the energy dissipated in the adaptive pneumatic fixture from the loading-test process, which can be exactly expressed as:

$$
\Delta U=\oint F d X
$$

Then the Loss-Coefficient $\eta$, the energy loss per radian divided by the maximum elastic-energy (also called as the total vibration energy throughout all frequencies), can be calculated as:

$$
\eta=\frac{\Delta U}{2 \pi U}=\frac{D}{2 \pi}=\frac{1}{Q} \approx 0.18 \text { (For the loading-test in Figure-11) }
$$

Where: $\quad F$ is the incremental loading-and-unloading force and $F_{\max }$ is the maximum loading-force from the loading-test-loop $(\mathrm{kN})$.

$X$ and $X_{\max }$ are the corresponding engine-casing surface deformation ( $\left.\mathrm{mm}\right)$.

$K_{3}$ is the averaged structural stiffness $(\mathrm{kN} / \mathrm{mm})$.

For a metallic thin-walled structure, its loading-tested loss-coefficient is generally in the range of $\eta \leq 0.01$ [21]. For a metallic thin-walled structure with plastic or polymeric damping-coats, such as the Constrained-Layer-Damper (CLD) [22], its loading-tested loss-coefficient is approximately in the range of $0.01 \leq \eta \leq 0.05$. As calculated with equation (11), the loading-tested loss-coefficient of the engine-casing with adaptive-fixture support, $\eta \approx 0.18$, which is more than 3.6 times higher than the engine-casing with CLD-damper and 18 times higher than the engine-casing without any damping 
applications. Obviously, this is a significant improvement on the dynamic performance of the thinwalled engine-casing. Practical machining (testing and massive-production) of similar thin-walled parts with varied-dimensions in a wide range and different hard-to-machine materials in real industrial environments proves this patented invention [15] to be a robust and reliable engineering solution.

\section{Summary}

As a summary of the whole paper, an ancient music instrument, the violin, and a very well developed dynamics theory [8], can be taken as a good example to simulate a dynamic machining system for thin-walled structure, as shown in Figure-12. From this comparison, another important principle of dynamic machining solutions can be well concluded, that is, taking the whole machining system including cutting-tool, work-piece, fixture and machine-tool as a mutually linked dynamic integrity and improving the weakest dynamic link for an optimal dynamic machining solution.

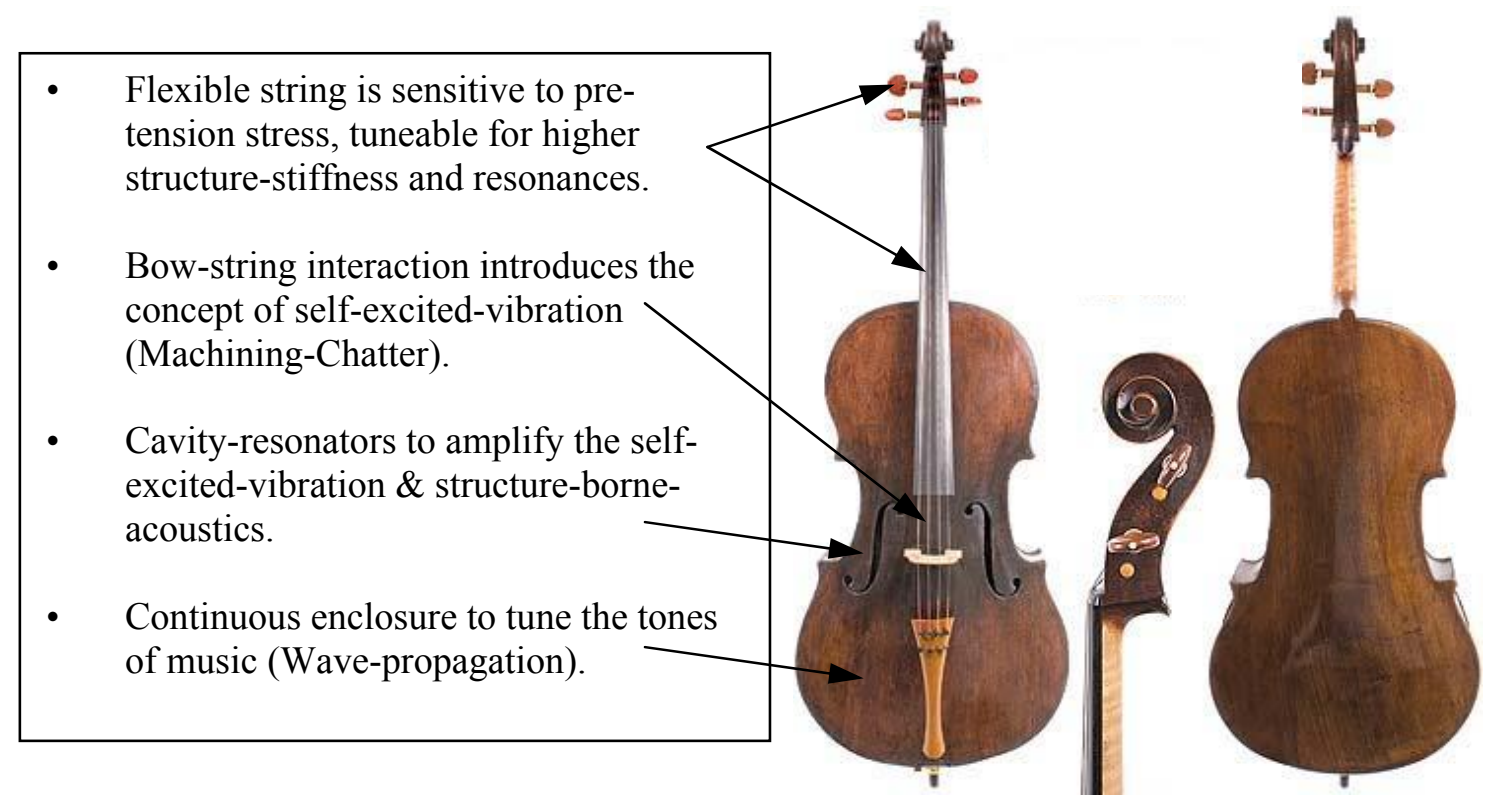

Figure-12. A simulative dynamic machining system: Dynamics of music instruments

(1) Flexible string is sensitive to pre-tension stress, tuneable for higher stiffness and resonances.

As shown in Figure-12, the flexible string is comparable to thin-walled flexible structures. Therefore, continuous modeling, identification and dynamic solutions are essential for it [8-14]. As the string is very sensitive to pre-tension stress (resonant frequency proportional to the square-root of pretension force [14]), naturally, pre-stress-tuning for a higher structure-stiffness and resonances could be a dynamic fixture solution for thin-walled structures.

(2) Bow-string interaction introduces the concept of self-excited-vibration.

The original definition of self-excited-vibration was defined by Lord Rayleigh, from his famous works by investigating the bow-string interaction in playing a violin [8]. This is the same workingmechanism of machining-chatter in advanced manufacturing, redefined in a more contemporary way by some contemporary scientists [1-2]. The main differences lie in the facts that, the contemporary scientists [1-3] are more focused on solving the machining chatter problem by improving the tooling dynamics, while the original finding [8] supplies a more profound theory including the whole dynamic system. 
(3) Cavity-resonator to amplify the self-excited-vibration and structure-borne acoustics.

Comparable to the violin dynamics, cavity-resonators in a dynamic machining system could be part of the work-piece, fixture and/or machine-tool itself. In most cases, the cavity-resonators amplify the self-excited-vibration and structure-borne acoustics, and make the machining process unstable. However, in some occasional cases, well designed cavity-resonators will disturb the existing structural acoustic-field and suppress the machining-induced vibration and noise, such as the well-known Helmholtz-resonators in controlling sound propagation in porous media [23].

(4) Continuous enclosure to tune the tones of music (Wave-propagation).

Existence of continuous enclosure or cavity in an elastic solid material is always a source of noise and acoustic-wave-propagation, which amplifies a well-tuned music from the violin or a badly tuned noise from the machining process. The above-discussed patented fixture [15] supplies a good solution for this example.

\section{References}

[1] G. Tlusty: Manufacturing processes and equipment. Prentice hall, Upper saddle river, New Jersey 07458, 1999.

[2] Y. Altintas: Manufacturing automation. Cambridge University Press, 2000.

[3] Z. Geng, K. Ridgway and S. Turner: Linear improvement of machining stability lobes and application in milling process prediction. Proceedings of the institution of mechanical engineers (IMechE), Part-B, Journal of engineering manufacturing, Vol. 221 (2007), pp369378.

[4] H. Goldstein: Classical mechanics. Addison-wesley, Reading MA, 1950.

[5] S. Timoshenko, D. H. Young and W. Weaver: Vibration problems in engineering. Wiley, 1974.

[6] G. B. Warburton: The dynamical behaviour of structures. Pergamon Press, Oxford, 1976.

[7] M. Geradin and D. Rixen: Mechanical vibrations - Theory and application to structural dynamics. John Wiley \& Sons, $2^{\text {nd }}$ edition, 1997.

[8] J. W. S. Rayleigh: The theory of Sound (2 volumes). Dover, New York, 1945.

[9] R. G. White and J. G. Walker: Noise and vibration. Ellis Horwood, Chichester, 1982.

[10] Diamler-Benz AG: Travelling-wave-motor: An electric drive system with future. TE/P 6705.2104.02.0791, 1991.

[11] H. Salleh and M. J. Brennan: Control of flexural waves on a beam using a vibration neutraliser: Effects of different attachment configurations. Journal of Sound and Vibration, 303 (2007), pp501-514.

[12] R. H. Lyon: Statistical Energy Analysis of dynamical systems: Theory and applications. The MIT Press, 1975.

[13] LMS Test.Lab manual: Estimation of modal parameters. Rev. 9A. Copyright LMS International 2009.

[14] J. P. Den Hartog: Mechanical Vibration. Mcgraw-Hill Book Company, 1968.

[15] Z. Geng: Adaptive design of pneumatic fixture for thin-walled shell/cylindrical components. UK-Patent: P112668GB (06/03/2007), International-Patent: WO2008107672 (15/09/2008), EU-Patent: PCT/GB2008000745 (09/10/2009), China-Patent: 200880007363.0 (07/09/2009), India-Patent: PCT/GB2008/000745 (Due-date: 06/03/2011), USA and Japan Patent applications in progress.

[16] L. Gaudiller and S. Bochard: Adaptive active control of flexible structures subject to rigid body displacements. Journal of Sound and Vibration, 283(2005), pp311-339.

[17] L. Zhang, Y. Xiang and G. W. Wei: Local differential quadrature for free vibration analysis of cylindrical shells with verious boundary conditions. International journal of mechanical science, 48 (2006), pp1126-1138.

[18] Sungsoo Na, Liviu Librescu and Jae Kyung Shim: Modelling and bending vibration control of non-uniform thin-walled rotating beams incorporating adaptive capabilities. International 
journal of mechanical science, 45 (2003), pp1347-1367.

[19] Z. Geng, A. A. Popov and D. J. Cole: Measurement, identification and modelling of damping in pneumatic tyres. International journal of mechanical science, Vol. 49 (2007), pp1077-1094.

[20] D. Cebon and M. F. Ashby: Materials selection for precision instruments. Measurement science and technology, Vol. 5 (1994), pp296-306.

[21] A. D. Nashif, D. Jones and J. P. henderson: Vibration damping. Wiley Interscience, 1985.

[22] G. R. Tomlison: The use of constrained layer damping in vibration control. International journal of mechanical science, Vol. 32 (1990), pp233-242.

[23] J. F. Allard: Propagation of sound in porous media. Elsevier Applied Science, London, 1993. 